\title{
INFORMAÇÃO CIENTÍFICA E TECNOLÓGICA: ANÁLISE DAS PUBLICAÇõé PERIÓdICAS DA ÁREA DA CIÊNCIA DA INFORMAÇÃO
}

\author{
SCIENTIFIC AND TECHNOLOGICAL INFORMATION: ANALYSIS OF PERIODIC \\ PUBLICATIONS OF INFORMATION SCIENCE
}

INFORMACIÓN CIENTÍFICA Y TECNOLÓGICA: ANÁLISIS DE LAS PUBLICACIONES PERIÓDICAS DEL ÁREA DE LA CIENCIA DE LA INFORMACIÓN

${ }^{1}$ Mayara Cintya do Nascimento Vasconcelos, ${ }^{1}$ Gabriela Belmont de Farias 'Universidade Federal do Ceará

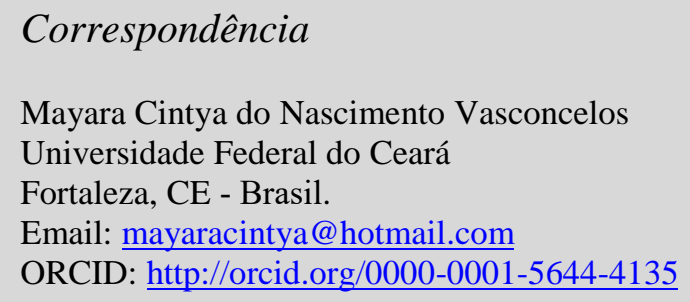

Submetido em: $31 / 03 / 2017$

Aceito em: 14/09/2017

Publicado em: 25/09/2017

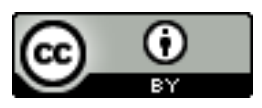

JITA: HA. Periodicals, Newspapers. 
RESUM0: A pesquisa analisa os artigos publicados em periódicos científicos nacionais da área da Ciência da Informação, classificados com Qualis A1, tendo como parâmetro o termo "informação científica e tecnológica". Apresenta conceitos acerca da informação científica e tecnológica e dos processos que envolvem seus usos, bem como a comunicação científica, fluxos informacionais e fontes de informação. A metodologia empregada consiste em estudo descritivo com abordagem quanti-qualitativa, com utilização das técnicas da bibliometria para medição de índices e da análise de conteúdo de Bardin. Foram analisados trinta e seis artigos, enquanto do qualitativo foram vinte e um resumos. Os resultados mostram que o periódico que mais publicou sobre o termo pesquisado foi "Informação e Sociedade", o ano com maior número de publicações foi 2016. A autoria múltipla foi uma tendência constatada nos artigos e as palavras-chave mais utilizadas foram: acesso livre, informação científica e tecnológica e preservação digital. Quanto ao conteúdo dos resumos foi possível identificá-los dentro de três categorias de análise, sendo elas: ciência da informação e processos que envolvem o uso da informação; produção, comunicação e avaliação da atividade científica e tecnológica; e desenvolvimento científico e tecnológico. Conclui-se que há uma tendência da literatura analisada em se voltar para o estudo da própria informação, de metodologias aplicadas em estudos e ainda de produtos e serviços da informação. Os resumos abordaram temas como repositório institucional, bibliometria, coautoria, gestão de revistas científicas e políticas de informação científica e tecnológica.

PALAVRAS-CHAVE: Informação científica. Informação tecnológica. Produção científica. Periódicos científicos.Comunicação científica.

ABSTRACT: The research analyzes the articles published in national scientific journals of the area of Information Science, classified with Qualis A1, having as parameter the term "scientific and technological information". It presents concepts about scientific and technological information and the processes that involve its uses, as well as scientific communication, information flows and sources of information. The methodology used is a descriptive study with a quantitative-qualitative approach, using bibliometrics techniques for index measurement and Bardin content analysis. Thirty - six papers were analyzed, while the qualitative ones were twenty - one abstracts. The results show that the most published journal about the term researched was "Information and Society", the year with the highest number of publications was 2016. Multiple authorship was a trend observed in the articles and the most used keywords were: free access, Scientific and technological information and digital preservation. As for the content of the abstracts, it was possible to identify them within three categories of analysis, namely: information science and processes involving the use of information; Production, communication and evaluation of scientific and technological activity; And scientific and technological development. It is concluded that there is a tendency of the literature analyzed to return to the study of the information itself, of methodologies applied in studies and also of information products and services. The abstracts addressed topics such as institutional repository, bibliometrics, coauthoring, management of scientific journals and scientific and technological information policies.

KEYWORDS: Scientific information. Technological information. Scientific production. Scientific journals. Scientific communication.

RESUMEN: La investigación analiza los artículos publicados en periódicos científicos nacionales del área de la Ciencia de la Información, clasificados con Qualis A1, teniendo como parámetro el término "información científica y tecnológica". Presenta conceptos acerca de la información científica y tecnológica y de los procesos que involucran sus usos, así como la comunicación científica, flujos informativos y fuentes de información. La metodología empleada consiste en un estudio descriptivo con abordaje cuantitativo, con la utilización de las técnicas de la bibliometría para medición de índices y del análisis de contenido de Bardin. Se analizaron treinta y seis artículos, mientras que el cualitativo fueron veintiún resúmenes. Los resultados muestran que el periódico que más publicó sobre el término investigado fue "Información y Sociedad", el año con mayor número de publicaciones fue 2016. La autoría múltiple fue una tendencia constatada en los artículos y las palabras clave más utilizadas fueron: acceso libre, información científica y tecnológica y preservación digital. En cuanto al contenido de los resúmenes fue posible identificarlos dentro de tres categorías de análisis, siendo ellas: ciencia de la información y procesos que involucra el uso de la información; producción, comunicación y evaluación de la actividad científica y tecnológica; y desarrollo científico y tecnológico. Se concluye que hay una tendencia de la literatura analizada en volverse hacia el estudio de la propia información, de metodologías aplicadas en estudios y aún de productos y servicios de la información. Los resúmenes abordaron temas como repositorio institucional, bibliometría, coautoria, gestión de revistas científicas y políticas de información científica y tecnológica.

PALABRAS Clave: Información científica. Información tecnológica. Producción científica. Periódicos científicos. Comunicación científica. 


\section{INTRODUÇÃ̃o}

A informação ocupa lugar de destaque em nossa sociedade, tanto nas relações pessoais quanto nas profissionais. Estamos constantemente usando-a para tomar decisões e resolver problemas cotidianos. Partindo para o campo científico e tecnológico é ainda mais expressiva a relevância que a informação apresenta para o desenvolvimento social e econômico de um país, sua inserção no processo de comunicação científica propicia a percepção de aspectos relevantes sobre o conhecimento científico e o conhecimento tecnológico colocando-a como base para a inovação. (MOURA; ROZADOS; CAREGNATO, 2006).

Sua constituição, comunicação, transferência e uso são estudados pela Ciência da Informação (CI) com o intuito de uma melhor compreensão sobre sua influência no processo de desenvolvimento econômico e social do Brasil. A questão da informação científica e tecnológica (ICT) é imprescindível para o desenvolvimento do país partindo de uma perspectiva econômica e social.

A comunicação da ICT se processa através de dois tipos de fontes de informação: as formais e as informais. As primeiras são representadas por publicações primárias, secundárias, terciárias e pelos serviços e ambiente de informação. A divulgação dessa informação foi estabelecida pelo sistema de comunicação científica, o qual vem se consolidando ao longo do tempo. As segundas são representadas por diferentes modalidades de intercâmbio interpessoal, como conversas, correspondência, telefonemas e reuniões de caráter restrito.

São importantes não só os investimentos nesse tipo de informação, bem como os estudos sobre suas características, produção e disponibilização. Analisar a produção científica e tecnológica é, portanto, fundamental para o campo da CI.

Diante das observações acima temos o interesse de apresentar a análise realizada, em artigos, publicados em periódicos científicos nacionais da área da CI classificados com Qualis A1. Tendo como ponto de partida a apreciação a respeito da expressividade da temática "informação científica e tecnológica" na CI bem como identificar os autores que estão publicando sobre essa temática. Para tanto identificamos os periódicos científicos nacionais classificados em 2015 com classificação A1; identificamos o quantitativo de publicações por ano, tipo de autoria, autores em evidência, quantidade de artigos por instituição e ocorrência de palavras-chave. E analisamos o conteúdo dos resumos dos artigos selecionados para identificar as abordagens temáticas.

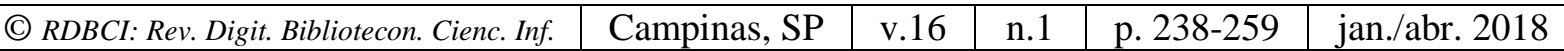




\section{INFORMAÇÃO E DESENVOLVIMENTO CIENTíFICO E TECNOLÓGICO}

$\mathrm{Na}$ atual conjuntura social observamos um crescente aumento no acesso e uso da informação, tornando-a relevante no processo de tomada de decisão em diversos campos do saber. A informação passa a ocupar lugar de destaque nas relações humanas e também passa a ser utilizada como matéria-prima no campo científico e tecnológico.

A informação, portanto, tem um valor estratégico em ambos os campos e representa "[...] mais que um meio de desenvolvimento econômico, a informação tem uma função paradigmática num mundo onde nada se produz aleatoriamente, a partir de uma ideia que brotou sem uma influência informativa externa." (SOUZA, p. 35, 1991).

O tratamento e a disponibilização das informações é fator crucial para o melhor aproveitamento e consequentemente otimização dos processos envolvidos com 0 desenvolvimento tanto científico como tecnológico. A informação científica pode ser conceituada como "[...] todo conhecimento que resulta - ou está relacionado com o resultado - de uma pesquisa científica.” (AGUIAR, p 10, 1991). A principal etapa da pesquisa científica é a divulgação dos resultados justamente pelo motivo da circulação das informações científicas que servirão de base para outras pesquisas e proporcionará o avanço da ciência, a divulgação também garante a autoria do sujeito que publicou. Corroborando com esse pensamento Mueller afirma que,

Para obter confiabilidade, além da utilização de uma rigorosa metodologia científica para a geração do conhecimento é importante que os resultados obtidos pelas pesquisas de um cientista sejam divulgados e submetidos ao julgamento de outros cientistas, seus pares. (MUELLER, 2000a, p. 21)

Esse processo de divulgação dos resultados e ponderação por parte de outros membros da comunidade científica garante ao pesquisador reconhecimento e validação de sua pesquisa.

As funções da informação científica foram elencadas por Aguiar (1991) sendo importante para a divulgação de conhecimento novo obtido por meio de pesquisa científica, validar o direito de prioridade intelectual, servir como input para uma nova pesquisa científica e explicitar a metodologia utilizada no projeto de pesquisa científica. Podemos inferir que a informação científica é ao mesmo tempo insumo e produto da pesquisa, ela agrega valores às informações anteriores e as transforma em algo novo:

A informação científica é, por isso, o conhecimento que constituiu, em um certo momento da evolução da ciência, um acréscimo ao entendimento universal então existente sobre algum fato ou fenômeno, tendo-se tornado disponível como resultado de uma pesquisa científica, ou seja, de um trabalho de investigação conduzido segundo o método científico. (AGUIAR, 1991, p.10).

Segundo Aguiar (1991, p. 11) a "[...] informação tecnológica é todo tipo de conhecimento relacionado com o modo de fazer um produto ou prestar um serviço, para colocá-lo no mercado [...]", tendo como funções: servir como matéria-prima para o

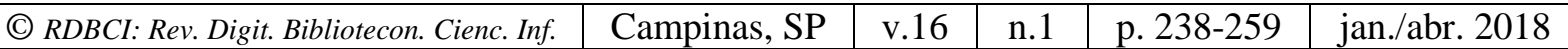


desenvolvimento de pesquisas tecnológicas, garantir o direito de propriedade industrial, divulgar tecnologias de domínio público, auxiliar o processo de gestão tecnológica, proporcionar acompanhamento e avaliação de tendências de desenvolvimento tecnológico, viabilizar a avaliação do impacto econômico, social e ambiental das tecnologias. (AGUIAR, 1991). Ela também serve como meio de confrontar informações anteriores, "A informação tecnológica leva os indivíduos a novas teorias, novas teses; ela traz experiências que foram desenvolvidas em outros ambientes que não o que se vivência, para que se possa avaliar, testar, analisar, criticar etc." (VALENTIM, 1997, p.27).

A informação tecnológica é insumo essencial para manter a competitividade no ambiente industrial. $\mathrm{O}$ processo de transformação da informação tecnológica em bem de consumo requer da indústria atenção especial e constante monitoramento, afirma que,

\begin{abstract}
Assim como a sociedade brasileira, o setor industrial brasileiro deve despertar definitivamente para a importância da informação, principalmente a tecnológica, tendo uma postura moderna com relação a ela e ter a conviç̧ão de que sem informação, principalmente a tecnológica, não haverá competitividade. (VALENTIM, 1997, p. 17)
\end{abstract}

As informações tecnológicas assumem na indústria papel crucial principalmente quando tratamos de Pesquisa e Desenvolvimento (P\&D). Roussel, Saad e Bohlin (1992) classificam P\&D em três tipos, incremental, radical e fundamental. A incremental tem como características a competente exploração do conhecimento técnico e científico, o baixo risco e a modesta recompensa. A radical permite a criação de novos conhecimentos para a empresa com um objetivo comercial característico, além de possuir um risco maior e elevada recompensa. A fundamental também permite a criação de novos conhecimentos para a empresa com o intuito de ampliar e aprofundar o entendimento da mesma possui alto risco e aplicabilidade incerta às necessidades comerciais.

Com relação ao campo científico Le Coadic (2004, p.26) elucida, "a informação é a seiva da ciência. sem informação, a ciência não pode se desenvolver e viver." Tal afirmação nos faz refletir sobre a informação como fator crucial para a continuidade das atividades científicas em todas suas etapas. No campo tecnológico é perceptível o valor que essa informação possui, sendo a informação tecnológica cada vez mais importante para a capacitação tecnológica das indústrias e necessária para subsidiar a tomada de decisões, visando à competitividade e desenpenhando papel fundamental no processo de P\&D. (VALENTIM, 1997; JANNUZZI, 2002).

A informação também é fator imprescindível para o desempenho econômico e seu processo de comunicação contribui para a eficiência das empresas, "Em termos econômicos, cada vez mais se reconhece que a informação (e sua efetiva comunicação) é o recurso mais importante para eficiência de qualquer indústria, processo de produção ou comércio." (FREIRE, 1991, p.21). É importante ressaltar que as informações científicas e tecnológicas

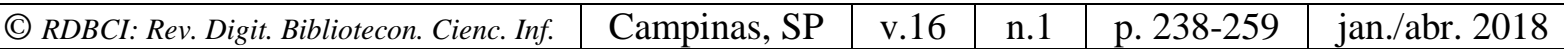


são complementares, o que corrobora com o pensamento de Le Coadic (2004) sobre a questão de a industrialização passar pela ciência e da ciência passar pela industrialização.

\section{CIÊNCIA DA INFORMAÇÃo, ORGANIZAÇÃ̃ E DIVULGAÇÃo DA INFORMAÇÃO CIENTÍFICA E TECNOLÓGICA}

Le Coadic (2004, p. 25) enuncia que a Ciência da Informação é "[...] uma ciência social rigorosa que se apoia em uma tecnologia também rigorosa" e ainda que "tem por objeto o estudo das propriedades gerais da informação (natureza, gênese, efeitos), e a análise de seus processos de construção, comunicação e uso.”.

O autor discute os três processos das informações científicas e técnicas: construção, comunicação e uso. No primeiro processo, a construção, é abordada a questão da relevância das atividades científicas e técnicas sendo elas "[...] o manancial de onde fluem os conhecimentos científicos e técnicos que se transformarão, depois de registrados, em informações científicas e técnicas." (LE COADIC, 2004, p.26). O amplo crescimento das atividades de pesquisa desencadeou num processo de valorização da informação onde o sistema de construção de conhecimentos se incorporou ao desenvolvimento econômico e social. Esse crescimento possibilitou a multiplicação do número de periódicos especializados e consequentemente o aumento da literatura científica, o que em tese, não significou o crescimento igualitário do ponto de vista qualitativo.

Como características do crescimento da informação o autor cita a ampliação dos setores onde se exerce esse conhecimento; o movimento de síntese e o desejo de unidade; o aparecimento de novos produtos, processos de produção, atividades e empresas; a introdução de novos produtos no mercado, o desenvolvimento de novos processos de produção e a criação de novas atividades e novas empresas. Diante de todo esse processo de crescimento temos os atores da construção dessa informação, a comunidade científica que em termos gerais "[...] é o grupo social formado por indivíduos cuja profissão é a pesquisa científica e tecnológica." (LE COADIC, 2004, p. 28), essa comunidade permite ao cientista que ele compartilhe seus resultados e como retorno dessa atividade ele obtém o reconhecimento de seus pares.

A comunicação no meio científico é estritamente importante para o desenvolvimento de pesquisas. De acordo com Le Coadic (2004, p. 32), "O papel da comunicação consiste em assegurar o intercâmbio de informações sobre os trabalhos em andamento, colocando os cientistas em contato entre si.". Essa comunicação pode ser classificada em informal, quando possui natureza mais pessoal, também abrange pesquisas não concluídas, comunicação de pesquisa em andamento etc, e formal, quando comporta publicações amplamente divulgadas como artigos publicados em periódicos científicos e livros. (MUELLER, 2000a). 
A evolução do campo científico está intrinsicamente ligada à sua comunicação, pois a partir desse processo os estudos realizados podem ser comparados, refutados e aperfeiçoados, permitindo um ciclo contínuo da informação. Sobre a importância dos sistemas de comunicação Targino (2000, p. 5) afirma,

Finalmente, qualquer que seja a ótica adotada para o estudo do desenvolvimento da ciência, a natureza dos sistemas de comunicação resulta vital para a ciência e está no âmago do método científico. Não há ciência sem comunicação. Não há comunicação sem informação.

A comunicação científica atua como mediadora no processo de troca de informações, permitindo assim a formação e continuidade de fluxos informacionais,

\begin{abstract}
Esta permite a troca de informações, donde se conclui que enquanto a informação é um produto, uma substância, uma matéria, a comunicação é um ato, um mecanismo, é o processo de intermediação que permite o intercâmbio de ideias entre os indivíduos. A comunicação é um fenômeno natural e intrínseco ao homem, variando de acordo com as características dos grupos nos quais e entre quais se efetiva. Como tal, o processo de comunicação pressupõe um estoque comum de elementos preexistentes - linguagem, expressões, códigos etc -, essencial para facilitar o fluxo informacional. (TARGINO, 2000, p. 10).
\end{abstract}

Um modelo famoso de comunicação científica é o que apresenta o processo de forma contínua, onde as atividades realizadas pelo pesquisador geram documentos como relatórios preliminares, comunicações de pesquisa em andamento. Ao término da pesquisa há apresentação em seminários, colóquios, conferências que produzem trabalhos escritos completos ou resumos que serão amplamente divulgados e servirão como referência para outras pesquisas. (GARVEY; GRIFFITH, 1972 apud MUELLER, 2000a).

A respeito do modelo Mueller (2000a, p. 30) ainda ressalta, “[...] é fácil perceber que a informação flui por muitos canais e que diferentes tipos de documentos são produzidos, cujas características variam conforme o estágio da pesquisa e tipo de público a que se destina e o objetivo de quem a comunica.", é interessante notar como a informação vai se modificando ao longo do fluxo de acordo com a finalidade definida pelo produtor dessa informação.

Os fluxos informacionais surgiram a partir da explosão quantitativa da informação e da diminuição do tempo de comunicação da mesma. Esses fluxos têm como características a circulação de relevantes quantidades de informação por unidade de tempo que rompem as fronteiras nacionais. A internacionalização do mercado da informação pode ser ilustrada pelos fluxos de dados, esses fluxos também permitem a compreensão do progressivo aumento do interesse pelo conhecimento conforme demanda, monitoramento da informação, inteligência competitiva e gestão do conhecimento. (LE COADIC, 2004).

A comunicação científica e os fluxos informacionais fazem uso de fontes de informação que são de suma importância para a continuidade e qualidade dos processos científicos e tecnológicos. As fontes de ICT contribuem para o êxito na pesquisa, desenvolvimento, inovação e atividades ligadas à ciência e tecnologia,

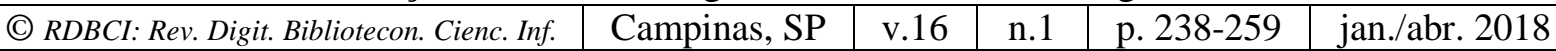


O uso efetivo da ICT ajuda a evitar a duplicação de trabalhos previamente realizados. Essa ação pode redundar na economia de tempo e de recursos materiais, humanos e financeiros. Além disso, e talvez o mais importante, pode servir de manancial de ideias ou para o desenvolvimento de uma ideia. A ICT, portanto, pode funcionar como uma valiosa fonte de inspiração e serendipidade para o aluno, professor, profissional ou pesquisador. (CUNHA, 2016, p.ix.).

As fontes de informação podem ser classificadas em: primárias, secundárias e terciárias. Os congressos e conferências, legislação, nomes e marcas comerciais, normas técnicas, patentes, periódicos, relatórios técnicos, teses e dissertações, são alguns exemplos de fontes primárias. Bancos de dados e bases de dados, bibliografias, biografias, catálogos de bibliotecas, dicionários, enciclopédias, feira e exposições, filmes e vídeos, imagens, livros, manuais são exemplos de fontes secundárias. Bibliografias de bibliografias e diretórios são exemplos de fontes terciárias. Queremos dar destaque aqui ao periódico como fonte primária de informação, pois é o um dos meios mais eficientes quando se trata do registro e divulgação de pesquisas, estudos originais e outros tipos de trabalho intelectual, "É o tipo de publicação primária considerada a mais atualizada e importante em ciência e tecnologia." (CUNHA, 2016, p.21.).

Os primeiros periódicos científicos surgiram na França e Inglaterra, respectivamente, e obtiveram boa aceitação por parte da comunidade científica, o que impulsionou o surgimento de outros periódicos em vários países da Europa, com a finalidade principal de divulgar as pesquisas que estavam sendo realizadas por seus membros." (MUELLER, 2000b, p. 75). As funções dos periódicos científicos de acordo com a Royal Society são elencadas por Mueller (2000b, p. 75-76),

a. comunicação formal dos resultados da pesquisa original para a comunidade científica e demais interessados

Essa era uma das funções originais do periódico, permanecendo praticamente inalterada até hoje;

b. preservação do conhecimento registrado

Em conjunto, os periódicos servem como arquivo das idéias e reflexões dos cientistas, dos resultados de suas pesquisas e observações sobre os fenômenos da natureza; a preservação e organização dos periódicos, nas bibliotecas do mundo todo, garantem a possibilidade de acesso aos conhecimentos registrados ao longo do tempo; tem sido uma das responsabilidades mais importantes dos bibliotecários;

c. estabelecimento da propriedade intelectual

Ao publicar seu artigo, tornando públicos os resultados de suas pesquisas, o autor registra formalmente a sua autoria, requerendo para si a prioridade na descoberta científica;

d. manutenção do padrão da qualidade da ciência

A publicação em periódicos que dispõem de um corpo de avaliadores respeitados confere a um artigo autoridade e confiabilidade, pois a aprovação dos especialistas representa a aprovação da comunidade científica; sem ela um pesquisador não consegue publicar seu artigo em periódicos respeitados; sem publicar não consegue reconhecimento pelo seu trabalho.

Com a evolução da tecnologia os periódicos ocuparam também o meio eletrônico sendo eles "[...] um meio de comunicação extremamente versátil e rápido, que permite a divulgação da pesquisa imediatamente após sua conclusão, ignorando barreiras geográficas 
para acesso [...], minimizando barreiras hierárquicas e permitindo a recuperação de informações de várias maneiras." (MUELLER, 2000b, p. 85).

\section{METODOLOGIA DA PESQUISA}

A pesquisa se enquadra em uma abordagem de métodos mistos já que além de elencar e descrever dados precisos sobre a produção científica buscou por meio da análise qualitativa levantar e responder questionamentos acerca da temática, relacionando o referencial teórico com o comportamento que a literatura apresentou. Creswell (2007, p. 35) salienta que,

[...] uma técnica de métodos mistos é aquela em que o pesquisador tende a basear as alegações de conhecimento em elementos pragmáticos (por exemplo, orientado para consequência, centrado no problema e pluralista). Essa técnica emprega estratégias de investigação que envolvem coleta de dados simultânea ou sequencial para melhor atender os problemas de pesquisa. A coleta de dados também envolve a obtenção tanto de informações numéricas (por exemplo, em instrumentos) como de informações de texto (por exemplo, em entrevistas), de forma que o banco de dados final represente tanto informações quantitativas como qualitativas.

A escolha por esse tipo de abordagem deu-se, principalmente, pelo cumprimento dos objetivos dessa pesquisa que consistem em elencar dados quantitativos da produção científica e ainda analisar o conteúdo dos resumos dos artigos, sendo os dois tipos de pesquisas complementares e importantes para o embasamento dos resultados. Para caracterizar os dados quantitativos usou-se a Bibliometria que consiste "[...] na aplicação de técnicas estatísticas e matemáticas para descrever aspectos da literatura e de outros meios de comunicação (análise quantitativa da informação) [...]. (ARAÚJO, 2006, p.12). A técnica utilizada na abordagem qualitativa foi a análise de conteúdo que compreende as etapas de pré-análise, exploração do material e tratamento dos resultados, inferência e interpretação.

Selecionamos três periódicos nacionais, na plataforma Sucupira, que se enquadravam como periódicos científicos, disponíveis online com classificação Qualis A1. São eles: Informação e Sociedade, Perspectivas em Ciência da Informação e Transiformação. Em seguida realizamos a busca no endereço eletrônico de cada periódico, com o seguinte termo de busca "informação científica e tecnológica" no campo "pesquisa" com todas as categorias incluídas pela necessidade de delimitar o corpus da análise.

Obtivemos como amostra 40 resultados, sendo excluídos do corpus da análise quatro dos resultados (Quadro 1) por não se enquadrarem como artigos.

QUADRO 1. Resultado das buscas em todos os periódicos

\begin{tabular}{|l|c|}
\hline \multicolumn{1}{|c|}{ Periódicos } & Resultados \\
\hline Informação e Sociedade & 16 \\
\hline Perspectivas em Ciência da Informação & 12 \\
\hline TransInformação & 8 \\
\hline \multicolumn{1}{|c|}{ TOTAL } & 36 \\
\hline
\end{tabular}

Fonte: Dados da pesquisa (2017).

\begin{tabular}{l|l|l|l} 
v.16 & n.1 & p. $238-259$
\end{tabular}


O periódico que apresentou mais publicações foi "Informação e Sociedade" com um total de 16 artigos, em seguida temos "Perspectivas em Ciência da Informação" com 12 artigos e "TransInformação" com 8 artigos.

\section{APRESENTAÇÃo E ANÁLISE DOS RESULTADOS}

A análise bibliométrica comportou trinta e seis artigos sendo dezesseis provenientes do periódico "Informação e Sociedade", doze do Periódico "Perspectivas em Ciência da Informação" e oito do periódico "Transinformação".

Uma das variáveis elencadas nos objetivos específicos deste trabalho versa sobre a quantidade de artigos publicados pelos periódicos ao longo dos anos, sendo o critério utilizado o primeiro ano em que um artigo com a temática foi recuperado e ano mais recente para fazer o recorte de tempo, de acordo com esse critério analisamos o período de 27 anos (1989 a 2016).

Os resultados mostram que o primeiro artigo recuperado com o termo aparece em 1989 no periódico "TransInformação", nenhum resultado foi encontrado nos anos de 1990, 1992 e 1993. No período de 1996 a 2002 a busca também não ofereceu resultados, em 2003 um artigo foi recuperado no periódico "Perspectivas em Ciência da Informação", já em 2004 e 2005 nenhuma publicação foi encontrada. De 2006 a 2016 foram encontrados resultados, sendo destaque os anos de 2007 com quatro publicações, 2010 com três, 2012 com quatro, 2015 com quatro e 2016 com cinco. 
Tabela 1. Ano versus quantidade de publicações por periódico

\begin{tabular}{cccccc}
\hline Ano & $\begin{array}{c}\text { Informação e } \\
\text { sociedade }\end{array}$ & $\begin{array}{c}\text { Perspectivas em } \\
\text { ciência da } \\
\text { informação }\end{array}$ & Transinformação & Total & $\%$ \\
\hline 1989 & 0 & 0 & 1 & 1 & 2,8 \\
1991 & 1 & 0 & 0 & 1 & 2,8 \\
1994 & 1 & 0 & 0 & 1 & 2,8 \\
1995 & 1 & 0 & 0 & 1 & 2,8 \\
2003 & 0 & 1 & 0 & 1 & 2,8 \\
2006 & 1 & 0 & 1 & 2 & 5,6 \\
2007 & 2 & 0 & 2 & 4 & 11,1 \\
2008 & 1 & 1 & 0 & 2 & 5,6 \\
2009 & 0 & 1 & 0 & 1 & 2,8 \\
2010 & 3 & 0 & 0 & 2 & 8,3 \\
2011 & 1 & 1 & 0 & 4 & 11,1 \\
2012 & 2 & 2 & 0 & 2 & 5,6 \\
2013 & 1 & 1 & 0 & 2 & 5,6 \\
2014 & 1 & 1 & 0 & 4 & 11,1 \\
2015 & 0 & 3 & 1 & 5 & 13,9 \\
2016 & 1 & 1 & 3 & 36 & 100 \\
\hline Total & 16 & 12 & 8 & & \\
\hline
\end{tabular}

Fonte: Dados da pesquisa (2017).

O gráfico a seguir permite uma melhor visualização da evolução das publicações dos periódicos ao longo dos anos, sendo a ápice de produção do periódico "Informação e Sociedade" em 2010 com três artigos, do periódico "Perspectivas em Ciência da Informação" em 2015 com três artigos e do periódico "TransInformação" em 2016 também com três artigos.

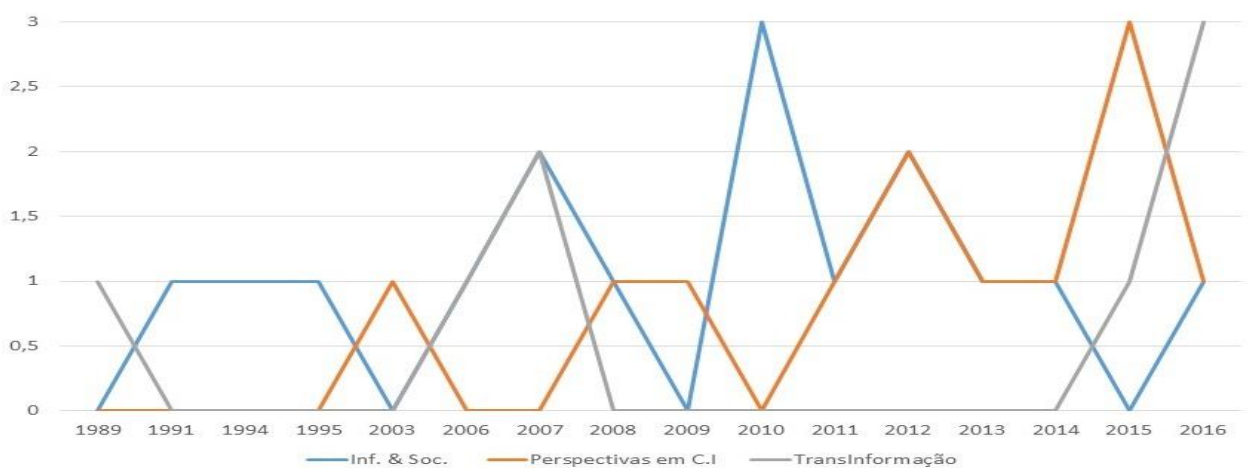

Gráfico 1. Distribuição do número de publicações dos periódicos por ano

Fonte: Dados da pesquisa (2017). 
Outro aspecto analisado foi o tipo de autoria dos artigos, os resultados mostraram que o tipo que prevaleceu foi a autoria múltipla de dois autores com 15 resultados dentre os artigos analisados, em segundo lugar vem a autoria única com 12 resultados, em terceiro a autoria múltipla de três autores com cinco resultados por fim a autoria múltipla de quatro autores com quatro resultados. A quantidade de artigos por tipo de autoria de cada periódico pode ser visualizada no seguinte gráfico,

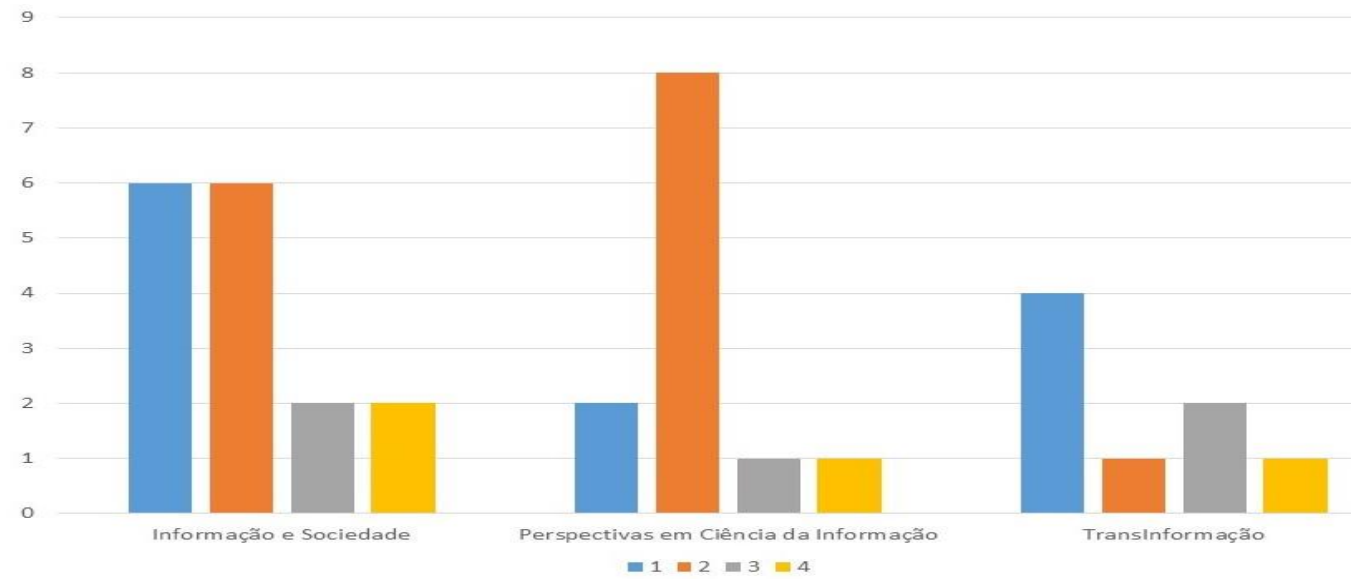

Gráfico 2. Quantidade de publicações por periódico x número de autores

Fonte: Dados da pesquisa (2017).

Os autores que mais obtiveram destaque foram elencados na tabela a seguir, o resultado engloba todos os tipos de autoria e foca no número de vezes em que cada autor aparece por artigo. Como destaque, temos um grupo formado por onze autores, sendo a autora que mais publicou artigos com o termo pesquisado Maria Cristina Soares Guimarães com cinco artigos e Alice Ferry de Moraes com três artigos.

Tabela 2. Autores em evidência

\begin{tabular}{lcc}
\hline \multicolumn{1}{c}{ Autores } & $\begin{array}{c}\text { Número de } \\
\text { artigos }\end{array}$ & $\%$ \\
\hline GUIMARÃES, Maria Cristina Soares & 5 & 13,9 \\
MORAES, Alice Ferry de & 3 & 8,3 \\
MOURA, Ana Maria Mielniczuk de & 2 & 5,6 \\
CENDÓN, Beatriz Valadares & 2 & 5,6 \\
SILVA, Cícera Henrique da & 2 & 5,6 \\
FLORES, Daniel & 2 & 5,6 \\
SILVA, Fábio Mascarenhas e & 2 & 5,6 \\
SANTOS, Henrique Machado dos & 2 & 5,6 \\
RODRIGUES, Jeorgina Gentil & 2 & 5,6 \\
TEIXEIRA, Robson da Silva & 2 & 5,6 \\
CAREGNATO, Sonia Elisa & 2 & 5,6 \\
\hline
\end{tabular}

Fonte: Dados da pesquisa (2017). 
A partir de uma análise do currículo Lattes das autoras, pontuamos considerações importantes com relação à temática estudada nesta pesquisa. Maria Cristina Soares Guimarães possui mestrado e doutorado em Ciência da Informação com títulos dos trabalhos de conclusão de curso respectivamente, "Avaliação em Ciência e Tecnologia: um estudo prospectivo em Química" e "Tecnologia como conhecimento: o público e o privado; o social e o econômico" é pesquisadora da Fundação Oswaldo Cruz (Fiocruz) o que justifica o fato de três dos artigos analisados serem estudos aplicados na instituição.

Outro ponto interessante é que Maria Cristina é coordenadora do Curso de Especialização lato sensu Informação Científica e Tecnológica em Saúde do Instituto de Comunicação e Informação Científica e Tecnologia em Saúde (Icict), seus temas de interesse incluem Acesso livre e informação científica e tecnológica em saúde, Avaliação em C\&T e Socialização da informação e estudos sociais da ciência e tecnologia o que esclarece o destaque da autora no estudo em questão. Alice Ferry de Moraes possui Especialização em Gerenciamento de Informação em C\&T, mestrado e doutorado em Ciência da Informação sendo seus trabalhos de conclusão respectivamente "Jornal - fonte de pesquisa. O uso da informação jornalística por pesquisadores para produção de conhecimento na Universidade" e "O uso de estratégias na transferência de informação nos vídeos em saúde", é servidora inativa da Fiocruz. É notável que ambas possuem interesse e produzem na área de C\&T, sendo parte de umas das instituições que mais atuam na divulgação da Informação científica e tecnológica.

A tabela três mostra as instituições com mais publicações em ordem decrescente. $\mathrm{O}$ levantamento leva em consideração o número de vezes em que instituição aparece nos artigos e envolve todos os tipos de autoria. A instituição que obteve maior destaque foi a Fiocruz com a aparição em nove artigos, em seguida UFRGS, UFMG, UFPB e UFRJ com três artigos, UNIRIO, UNESP, UEL, UFSM e UFSCAR com dois artigos, as outras instituições apresentaram um (artigo cada, em um dos artigos não foi possível identificar a instituição). 
Tabela 3. Quantidade de artigos por instituição

\begin{tabular}{ccc}
\hline Instituições & Artigos & $\%$ \\
\hline FIOCRUZ & 9 & 25 \\
UFRGS,UFMG,UFPB, & 3 & 8,3 \\
UFRJ & & \\
UNIRIO,UNESP,UEL & 2 & 5,6 \\
UFSM,UFSCAR & & \\
IFSP,USP,UESB & & \\
IBICT,PUC & & \\
CAMPINAS,UFPE & & 2,8 \\
UNICAMP,UFPR & 1 & \\
UFSC,PUC MINAS, & & \\
CIEB,UFPI,UNB & & \\
CEFET/RJ,UFABC & & \\
Sem identificação & 1 & \\
\hline
\end{tabular}

Fonte: Dados da pesquisa (2017).

A predominância de publicações da Fundação Oswaldo Cruz pode ser explicada pela notável relevância da instituição no país quando se trata da geração e difusão do conhecimento científico e tecnológico premissa essa que podemos encontrar na missão da fundação que consiste em, "Promover a saúde e o desenvolvimento social, gerar e difundir conhecimento científico e tecnológico, ser um agente da cidadania. Estes são os conceitos que pautam a atuação da Fundação Oswaldo Cruz (Fiocruz), vinculada ao Ministério da Saúde, a mais destacada instituição de ciência e tecnologia em saúde da América Latina." (FIOCRUZ, 2016).

Outra variável analisada foi a ocorrência de palavras-chave nos artigos, dos artigos recuperados quatro não apresentaram palavras-chave foram eles: "Política nacional de informação científica e tecnológica: necessidade versus realidade", "Ciência da Informação: uma ciência para a informação científica e tecnológica? ", "Educação e tecnologia: desenlaces/(entre)laços na aventura de (in)formar o homem - uma discussão possível?" e "Políticas e programas de informação e documentação da Unesco e fontes para seu estudo". $\mathrm{Na}$ totalidade obtivemos 120 palavras-chave, optamos por incluir na tabela as que se repetiam pelo menos uma vez, a palavra-chave que obteve maior destaque foi "Acesso livre", seguida de "Informação científica e tecnológica", "Preservação digital", "Produção científica", "Informação Científica", "Ciência da Informação", "Cientometria", "Coautoria", "Documentos arquivísticos digitais", "Interação C\&T", "Patente", "Plataforma lattes", "Recuperação da Informação", "Repositório institucional” e "Representação da Informação". 
Tabela 4. Ocorrência de palavras-chave

\begin{tabular}{lcc}
\hline \multicolumn{1}{c}{ Palavra-chave } & Repetições & $\%$ \\
\hline Acesso livre & 4 & 3,3 \\
Informação científica e tecnológica & 3 & 2,5 \\
Preservação digital & 3 & 2,5 \\
Produção científica & 2 & 1,7 \\
Informação científica & 2 & 1,7 \\
Ciência da Informação & 2 & 1,7 \\
Cientometria & 2 & 1,7 \\
Coautoria & 2 & 1,7 \\
Documentos arquivísticos digitais & 2 & 1,7 \\
Interação C\&T & 2 & 1,7 \\
Patente & 2 & 1,7 \\
Plataforma lattes & 2 & 1,7 \\
Recuperação da Informação & 2 & 1,7 \\
Repositório institucional & 2 & 1,7 \\
Representação da Informação & 2 & 1,7 \\
\hline
\end{tabular}

Fonte: Dados da pesquisa (2017).

As palavras-chave elencadas nos dão uma dimensão do conteúdo dos artigos. A predominância do termo "Acesso livre", por exemplo, indica certa preocupação com a democratização da informação científica e tecnológica nos artigos. Assim como "Produção científica", "Cientometria", "Coautoria", "Recuperação da informação" e "Representação da informação" indicam a realização de estudos do uso dessa informação. Para melhor compreender essas relações foi necessário analisar, além das palavras-chave, o conteúdo dos resumos dos artigos classificando-os em categorias de análise.

\subsection{Representações do conteúdo dos artigos}

Para a análise de conteúdo, no momento da pré-análise, optamos pela exclusão de alguns artigos pelo motivo de seus conteúdos fugirem da temática abordada neste trabalho. $\mathrm{O}$ resultado desse processo foi de 21 resumos de artigos para o corpus da análise de conteúdo.

As categorias de análise foram escolhidas a partir do conteúdo do referencial teóricoconceitual e da leitura dos resumos e palavras-chave dos artigos, o quadro a seguir mostrar a progressão das categorias analisadas. 
Quadro 2. Progressão das categorias de análise

\begin{tabular}{|c|c|c|}
\hline \multicolumn{3}{|c|}{ Progressão das categorias } \\
\hline INICIAIS & INTERMEDIÁRIAS & FINAIS \\
\hline 1. Representação da informação & \multirow{3}{*}{$\begin{array}{l}\text { I. Tratamento e gestão } \\
\text { da informação }\end{array}$} & \multirow{6}{*}{$\begin{array}{l}\text { I. Ciência da informação e } \\
\text { processos que envolvem o uso } \\
\text { da informação }\end{array}$} \\
\hline 2. Recuperação da informação & & \\
\hline 3. Gestão da informação & & \\
\hline 4. Disseminação da informação & \multirow{3}{*}{$\begin{array}{l}\text { II. Disponibilização e } \\
\text { monitoramento da } \\
\text { informação }\end{array}$} & \\
\hline 5. Acesso à informação & & \\
\hline 6. Monitoramento da informação & & \\
\hline 7. Produção científica e tecnológica & \multirow{2}{*}{$\begin{array}{c}\text { III. Produção e } \\
\text { comunicação científica }\end{array}$} & \multirow{5}{*}{$\begin{array}{c}\text { II. Produção, comunicação e } \\
\text { avaliação da atividade científica } \\
\text { e tecnológica }\end{array}$} \\
\hline 8. Comunicação Científica & & \\
\hline 9. Avaliação da produção científica & \multirow{3}{*}{$\begin{array}{l}\text { IV. Métodos de análise } \\
\text { da produção científica }\end{array}$} & \\
\hline 10. Bibliometria & & \\
\hline 11. Cientometria & & \\
\hline 12. Interação entre $C \& T$ & \multirow{3}{*}{$\begin{array}{l}\text { V. Informação como } \\
\text { insumo para o } \\
\text { desenvolvimento } \\
\text { tecnológico e } \\
\text { científico }\end{array}$} & \multirow{5}{*}{$\begin{array}{l}\text { III. Desenvolvimento científico } \\
\text { e tecnológico }\end{array}$} \\
\hline 13. Cultura científica e tecnológica & & \\
\hline 14. Inovação Científica e tecnológica & & \\
\hline 15. Programas de informação & \multirow{2}{*}{$\begin{array}{c}\text { VI. Políticas e } \\
\text { programas para C\&T }\end{array}$} & \\
\hline $\begin{array}{l}\text { 16. Políticas para a informação científica } \\
\text { e tecnológica }\end{array}$ & & \\
\hline
\end{tabular}

Fonte: Dados da pesquisa (2017)

A primeira categoria de análise "Ciência da informação e processos que envolvem o uso da informação" tem como objetivo agrupar artigos que contenham em seus resumos e palavras-chave os processos de representação, recuperação, gestão, disseminação, acesso e monitoramento da informação. Para melhor compreensão no corpo do texto de citações extraídas dos resumos, enumeramos os artigos em ordem crescente. Nesta categoria foram analisados sete artigos, os quais são enumerados de 1 a 7 .

Quadro 3. Categoria de análise I

\begin{tabular}{|c|c|}
\hline \multicolumn{2}{|c|}{ I. Ciência da Informação e processos que envolvem o uso da informação } \\
\hline \multirow{7}{*}{$\begin{array}{c}\text { Títulos } \\
\text { Dos } \\
\text { Artigos }\end{array}$} & A informação científica e tecnológica e os serviços de informação (1) \\
\hline & $\begin{array}{l}\text { As contribuições do design da informação para a democratização do acesso à informação } \\
\text { de bulas de medicamentos no Brasil (2) }\end{array}$ \\
\hline & $\begin{array}{l}\text { Monitoramento da informação na sociedade de risco: o caso da pandemia de gripe aviária } \\
\text { (3) }\end{array}$ \\
\hline & $\begin{array}{l}\text { Padrão de metadados pra obras raras na web: um estudo exploratório na Fundação } \\
\text { Oswaldo Cruz (4) }\end{array}$ \\
\hline & Utilização de Web Surveys para estudos de uso (5) \\
\hline & A utilização de boletim eletrônico no setor de referência: um estudo de caso (6) \\
\hline & $\begin{array}{l}\text { Organização da informação em sistemas eletrônicos abertos de informação científica \& } \\
\text { tecnológica: análise da Plataforma Lattes (7) }\end{array}$ \\
\hline
\end{tabular}

Fonte: Dados da pesquisa (2017).

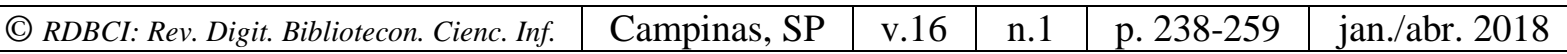


Um dos artigos faz uma abordagem acerca dos conceitos de informação científica, tecnológica e para negócios e os relaciona com os serviços de referência e informação fazendo uma comparação entre o Brasil e outros países, como ressaltado no trecho, "Enfatiza aspectos do serviço de informação tecnológica, tanto em países desenvolvidos quanto no Brasil. " (1). Um aspecto bastante abordado nos resumos é o acesso livre à informação científica e tecnológica o que fica elucidado em alguns trechos, "[...] apresentar a área de Design da informação para demonstrar como os aportes desta tecnociência podem contribuir para a democratização da informação, particularmente no acesso da informação técnicocientifica do setor da saúde." (2); "Discussão, avaliação e apresentação de parâmetros para a organização da informação científica e tecnológica brasileira, enfocando os problemas do acesso à informação em sistemas abertos” (7).

O artigo sobre Design da Informação mostra por meio da análise de bulas de medicamentos a importância dessa área no acesso à informação técnico-científica do setor da saúde. O artigo objetiva fazer uma apresentação da área e elucida sua contribuição para a democratização da informação. $\mathrm{O}$ artigo sobre sistemas abertos dá ênfase nos problemas enfrentados no acesso da informação científica e tecnológica. Promove discussão, avaliação e apresentação de parâmetros para a organização dessa informação. $\mathrm{O}$ estudo exploratório foi aplicado na Plataforma Lattes e tem por conclusão que a natureza aberta do sistema afeta a consistência dos dados na recuperação da informação, o artigo também apresenta sugestões para aprimorar o sistema.

Em um dos resumos há destaque para a memória científica da Fiocruz, apresentando-a como fonte de informação no campo da Ciência da Informação. O artigo apresenta proposta de um padrão de metadados para descrever obras raras no repositório institucional. Demanda essa que surgiu da dificuldade na gestão dos documentos analógicos e digitais. Os artigos também abordam a aplicação de métodos e a criação de serviços de informação, um dos artigos apresenta o método do Web Surveys aplicado à uma pesquisa sobre o uso do portal de periódicos da Capes. Os questionários da pesquisa foram enviados para aproximadamente 15 mil docentes distribuídos em 17 universidades federais. Apresenta ainda as dificuldades enfrentadas na aplicação do método e os resultados preliminares da pesquisa. Outro artigo apresenta estudo de caso em uma universidade no setor de referência de uma biblioteca do curso de física de uma universidade, a elaboração de um boletim eletrônico é apresentada como proposta para a disseminação da informação em universidades públicas brasileiras.

Há ênfase nos processos de representação da informação e sua posterior recuperação, um dos artigos ressalta o processo de categorização de documentos analisando as dificuldades e resultados em dois processos, um baseado no uso de palavras-chave e o outro no uso de citações para representar documentos. Dois experimentos foram realizados um com algoritmo de categorização baseado em palavras-chave outro com categorias utilizando redes neurais artificiais, detalha ainda a metodologia utilizada nos experimentos, ressalta a importância da pesquisa para os estudos de representação de documentos e recuperação da informação.

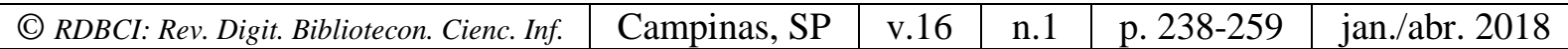


A segunda categoria de análise, "Produção, comunicação e avaliação da atividade científica e tecnológica", tem como objetivo agrupar os artigos que apresentaram em seus resumos temas como produção científica e tecnológica, comunicação científica, avaliação da produção científica, bibliometria e cientometria. Essa categoria incorporou oito artigos do total do corpus da análise enumerados de 8 a 15.

Quadro 4. Categoria de análise II

\begin{tabular}{|c|l|}
\hline II. Produção, comunicação e avaliação da atividade científica e tecnológica \\
\hline \multirow{4}{*}{$\begin{array}{c}\text { Títulos } \\
\text { Dos }\end{array}$} & $\begin{array}{l}\text { Co-classificação entre artigos e patentes: um estudo da interação entre C\&T na } \\
\text { Biotecnologia Brasileira (8) }\end{array}$ \\
\cline { 2 - 3 } Artigos & Experiências relacionadas ao levantamento de teses e dissertações (9) \\
\cline { 2 - 3 } & Repositório institucional da saúde: a experiência da Fundação Oswaldo Cruz (10) \\
\cline { 2 - 3 } & $\begin{array}{l}\text { Bo-autoria em artigos e patentes: um estudo da interação entre a produção científica e } \\
\text { tecnológica (12) }\end{array}$ \\
\cline { 2 - 3 } & Modelos de gestão de revistas científicas: uma discussão necessária (13) \\
\cline { 2 - 3 } & Exploração da Plataforma Lattes por assunto: proposta de metodologia (14) \\
\cline { 2 - 3 } & Terminologia como indicador qualitativo (15) \\
\hline
\end{tabular}

Fonte: Dados da pesquisa, (2017).

Os resumos contidos na categoria II destacam a produção científica e tecnológica, a clara interação entre os dois tipos de informação e ainda as formas de avaliar essa produção por meio da bibliometria, essa tendência apresentada nos resumos fica clara nos seguintes trechos, "Analisa a interação entre ciência e tecnologia (C\&T) a partir de um enfoque cientométrico, utilizando a técnica de co-classificação." (8); "Analisa a interação entre a produção científica e tecnológica dos pesquisadores que depositaram patentes na área da Biotecnologia" (12); "Para complementar a pesquisa, foi realizada uma pesquisa na plataforma Lattes, considerada como mais uma alternativa para o levantamento da produção científica e tecnológica." (9); “[...] com o objetivo de garantir a preservação da memória da produção científica e técnica do Icict e sua ampliação na perspectiva de promover o acesso livre e irrestrito da produção intelectual da Fiocruz." (10); "A Plataforma Lattes, no Brasil, é um relevante sistema de informação curricular que permite o registro da produção científica e tecnológica de cada pesquisador." (14).

A terceira categoria de análise, "Desenvolvimento científico e tecnológico", tem como objetivo comportar artigos que em seus resumos apresentavam temas como interação entre C\&T, cultura científica e tecnológica, inovação científica e tecnológica, programas de informação, políticas para a informação científica e tecnológica. Os artigos dessa categoria foram enumerados de 16 a 21. 
Quadro 5. Categoria de análise III

\begin{tabular}{|c|l|}
\hline \multicolumn{4}{|l|}{ III. Desenvolvimento científico e tecnológico } \\
\hline \multirow{4}{*}{ Títulos } & Informação e inovação na vacina da peste da Manqueira (16) \\
\cline { 2 - 3 } dos & Política nacional de informação científica e tecnológica: necessidade versus realidade (17) \\
\cline { 2 - 3 } artigos & Políticas e programas de informação e documentação da UNESCO e fontes para seu estudo \\
\cline { 2 - 3 } & Informação ambiental no Brasil: para quê e para quem (19) \\
\cline { 2 - 3 } & O óbvio da informação científica: acesso e uso (20) \\
\cline { 2 - 3 } & Patente gera patente? (21) \\
\hline
\end{tabular}

Fonte: Dados da pesquisa (2017).

Os resumos dessa categoria corroboram com o pensamento de Aguiar (1991) e Valentim (1997) que veem a informação científica e tecnológica como integrante de processos que geram pesquisas no meio científico e produtos no meio tecnológico. Podemos destacar trechos importantes nos resumos que sintetizam a ideia da informação como o insumo de processos que levam à vantagem no campo da inovação e do desenvolvimento científico e tecnológico, "[...] a informação como elemento estratégico para o processo de inovação científica e tecnológica." (16), "O texto aborda o acesso e o uso da informação científica, a partir da sua concepção e função para o avanço científico e tecnológico dos países. " (20). Os trechos destacados elucidam a crucial participação da informação nas atividades de cunho científico e tecnológico, colocando-a como centro desses processos. Os resumos também abordam a questão do acesso livre à informação científica e do fluxo informacional dessa informação.

Parte dos resumos foca na questão das políticas e programas e ainda levanta-se a questão da necessidade de uma política nacional de informação científica e tecnológica ressaltada no seguinte trecho, "Tenta-se vislumbrar a tendência atual da ICT - Informação Científica e tecnológica no Brasil, a partir de sua necessidade contraposta às implicações da sua conformação na realidade." (17). Também é feita a indicação de fontes de informação em atividades de programas no campo científico e tecnológico. Um tipo específico de informação foi abordado em um dos artigos, a informação ambiental. Nele é apontado a questão da difusão da informação, os profissionais da informação e o desenvolvimento autossustentável do Brasil. Um dos resumos apresenta como resultado da pesquisa o contraste no nosso país na utilização da patente nos campos da inovação e tecnologia, (21) "[...] a patente não se apresenta como informação necessária para a produção de tecnologia e inovação", o que leva o autor a constatar a necessidade de realizar ações para transferir e transformar patente em conhecimento. 


\section{CONCLUSÃo}

O estudo em questão abordou assuntos pertinentes quanto ao universo da informação científica e tecnológica e da produção científica. A descrição e análise dos dados apresentados permitiu uma maior compreensão da literatura sobre a temática, ainda que o recorte da pesquisa tenha englobado apenas parte desse ambiente. Os objetivos elencados foram alcançados, já que analisamos a literatura sobre o termo "informação científica e tecnológica" em três (3) periódicos Qualis A1 que estão incluídos no âmbito da Ciência da Informação. Para o cumprimento dos objetivos realizamos levantamento bibliográfico acerca da temática, trazendo conceitos e fatos importantes para a compreensão de modo geral. Descrevemos aspectos quantitativos do corpus da análise por meio de tabelas e gráficos e descobrimos as variáveis: quantidade de publicações por ano, tendo os anos de 2016, 2015, 2012, e 2007 como destaque de produção.

A autoria múltipla como tendência. As autoras Maria Cristina Soares e Alice Ferry de Moraes como destaque na produção, a instituição com maior destaque dentro da produção foi a Fundação Oswaldo Cruz e as palavras-chave mais usadas foram acesso livre, informação científica e tecnológica e preservação digital. Analisamos também o currículo Lattes das autoras mais produtivas o que nos permitiu concluir que suas formações e pesquisas estão voltadas para a área da Ciência e Tecnologia.

Os resumos dos artigos selecionados para a análise de conteúdo mostraram a tendência da literatura em se voltar para o estudo da própria informação, de metodologias aplicadas em estudos e ainda de produtos e serviços da informação. A análise da própria produção também foi um fator constatado nos resumos que abordaram temas como levantamento de teses e dissertações, repositório institucional, bibliometria, coautoria e gestão de revistas científicas. E ainda, constatamos a preocupação com as políticas de informação científica e tecnológica e suas contribuições para o desenvolvimento científico e tecnológico.

A pesquisa pretendeu, por meio de um recorte na literatura, entender os aspectos da ICT apresentada no conteúdo dos resumos de artigos que estão veiculados no principal meio de comunicação científica. Os dados quantitativos auxiliaram o entendimento e foram complementares e de fundamental importância para a conclusão do estudo. Queremos salientar que o estudo não pretendeu esvaziar as discussões sobre a temática ou levantar de forma exaustiva conteúdos e dados. Esperamos que assim como toda comunicação científica esse estudo sirva de insumo para o desencadeamento de outras pesquisas mais aprofundadas, contribuindo assim para o fluxo da informação e para o avanço da ciência.

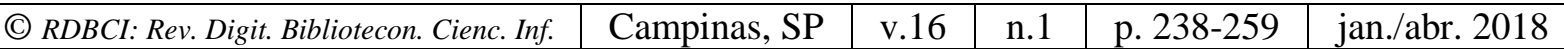




\section{REFERÊENCIAS}

AGUIAR, Afrânio Carvalho. Informação e atividades de desenvolvimento científico, tecnológico e industrial: tipologia proposta com base em análise funcional. Ciência da Informação, Brasília, v. 20, n.1, p. 7-15, jan./jun. 1991. Disponível em: <http://revista.ibict.br/ciinf/article/view/409>. Acesso em: 29 ago. 2016.

ARAÚJO, Carlos Alberto. Bibliometria: evolução histórica e questões atuais. Em Questão, Porto Alegre, v. 12, n. 1, p. 11-32, jan. /jun. 2006. Disponível em: < http://www.revistas.univerciencia.org/index.php/revistaemquestao/article/view/3707>. Acesso em: 3 nov. 2016.

CRESWELL, John W. Projeto de pesquisa: métodos qualitativo, quantitativo e misto. 2. ed. Porto Alegre: Artmed, 2007.

CUNHA, Murilo Bastos da. Para saber mais: fontes de informação em ciência e tecnologia. 2. ed. Brasília: Briquet de Lemos, 2016.

FIOCRUZ. Fundação Oswaldo Cruz. Rio de Janeiro. Disponível em: < http://www.portal.fiocruz.br/pt-br/content/funda\%C3\%A7\%A3o>. Acesso em: 3 nov. 2016.

FREIRE, Isa Maria. Barreiras na comunicação da informação tecnológica. Ciência da Informação, Brasília, v. 20, n.1, p. 51-54, jan./jun. 1991. Disponível em: <http://revista.ibict.br/ciinf/article/view/416/416>. Acesso em: 8 set. 2016.

JANNUZZI, Celeste Aída. Informação tecnológica e para negócios no Brasil: conceitos e terminologias. São Paulo: Alínea, 2002.

LE COADIC, Yves-François. A ciência da informação. 2. ed. Brasília: Briquet de Lemos, 2004.

MOURA, Ana Maria Mielniczuk de; ROZADOS, Helen Beatriz Frota; CAREGNATO, Sônia Elisa. Interações entre ciência e tecnologia: análise da produção intelectual dos pesquisadores-inventores da primeira carta-patente da UFRGS. Encontros Biblio, Florianópolis, v. 22, n. 2, 2006. Disponível em: <http://www.redalyc.org/articulo.oa?id=14702202>. Acesso em: 15 mar. 2017.

MUELLER, Suzana Pinheiro Machado. A ciência, o sistema de comunicação científico e a literatura científica. In: CAMPELLO, Bernadete Santos; CENDÓN, Beatriz Valadares; KREMER, Jeannette Marguerite (org.). Fontes de informação para pesquisadores e profissionais. Belo horizonte: Editora UFMG, 2000a, p. 21-34.

MUELLER, Suzana Pinheiro Machado. O periódico científico. In: CAMPELLO, Bernadete Santos; CENDÓN, Beatriz Valadares; KREMER, Jeannette Marguerite (org.). Fontes de informação para pesquisadores e profissionais. Belo horizonte: Editora UFMG, 2000b, p. 73-95. 
ROUSSEL, Philip A.; SAAD, Kamal N.; BOHLIN, Nils. Pesquisa \& desenvolvimento: como integrar P\&D ao plano estratégico e operacional das empresas como fator de produtividade e competitividade. São Paulo: Makron Books, 1992.

SOUZA, Francisco das Chagas. Uso da informação na indústria como paradigma para o desenvolvimento econômico. Ciência da Informação, Brasília, v. 20, n.1, p. 34-36, jan./jun. 1991. Disponível em:<http://revista.ibict.br/ciinf/article/view/413>. Acesso em: 29 ago. 2016.

TARGINO, Maria das Graças. Comunicação científica: uma revisão de seus elementos básicos. Informação e Sociedade: Estudos, João Pessoa, v. 10, n. 2, 2000. Disponível em: < http://www.ies.ufpb.br/ojs/index.php/ies/article/view/326>. Acesso em: 3 nov. 2016.

VALENTIM, Marta Lígia Pomim. O custo da informação tecnológica. São Paulo: Polis; APB, 1997.
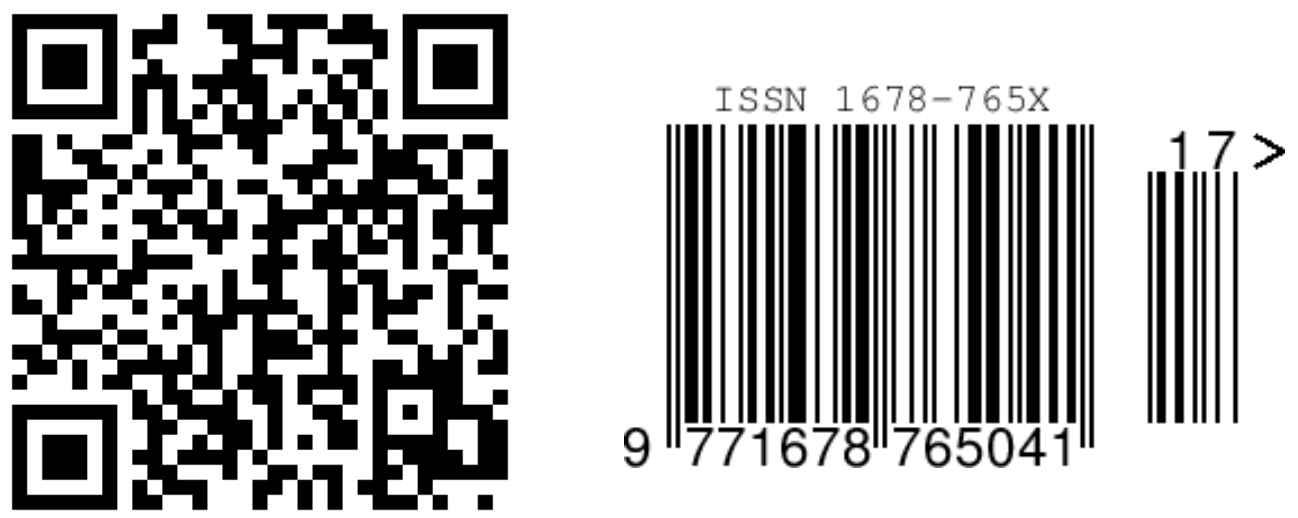